\title{
Utility of endoscopic therapy in the management of Boerhaave syndrome
}

Authors

Institutions
K. J. Dickinson ${ }^{1}$, N. Buttar ${ }^{2}$, L. M. Wong Kee Song ${ }^{2}$, C. J. Gostout ${ }^{2}$, S. D. Cassivi ${ }^{1}$, M. S. Allen ${ }^{1}$, F. C. Nichols ${ }^{1}$, K. R. Shen ${ }^{1}$, D. A. Wigle ${ }^{1}$, S. H. Blackmon ${ }^{1}$

${ }^{1}$ Division of General Thoracic Surgery, Mayo Clinic, Rochester, MN, USA

${ }^{2}$ Division of Gastroenterology, Mayo Clinic, Rochester, MN, USA submitted

18. September 2015 accepted after revision 22. August 2016

\section{Bibliography \\ DOI http://dx.doi.org/ \\ 10.1055/s-0042-117215 \\ Published online: 8.11.2016 \\ Endoscopy International Open \\ 2016; 04: E1146-E1150 \\ (c) Georg Thieme Verlag KG \\ Stuttgart · New York \\ E-ISSN 2196-9736}

Corresponding author

Shanda H. Blackmon, MD MPH

Division of General Thoracic Surgery

Mayo Clinic

200 First St, SW

Rochester

MN 55905

USA

Fax: +1-507-284-0058

Blackmon.Shanda@mayo.edu
Background/aims: The optimal intervention for Boerhaave perforation has not been determined. Options include surgical repair with/without a pedicled muscle flap, $\mathrm{T}$ tube placement, esophageal resection or diversion, or an endoscopic approach. All management strategies require adequate drainage and nutritional support. Our aim was to evaluate outcomes following Boerhaave perforation treated with surgery, endoscopic therapy, or both.

Patients and methods: We performed a 10-year review of our prospectively maintained databases of adult patients with Boerhaave perforations. We documented clinical presentation, extent of injury, primary intervention, "salvage" treatment (any treatment for persistent leak), and outcome. Results were analyzed using the Fisher's exact and Kruskal-Wallis tests.

Results: Between October 2004 and October 2014, 235 patients presented with esophageal leak/fistula with 17 Boerhaave perforations. Median age was 68 years. Median length of perforation was $1.25 \mathrm{~cm}$ (range $0.8-5 \mathrm{~cm}$ ). Four patients presented with systemic sepsis (two treated with

\section{Background and aims}

$\nabla$

The current practice of most thoracic surgeons who discover that a patient has an esophageal perforation involves a number of options. These include primary surgical repair (when possible) reinforced with a vascularized, pedicle-type muscle flap, nutritional support, and drainage. Alternative strategies include endoscopic therapies such as clipping, stenting or a combination thereof, $\mathrm{T}$ tube drainage and, in certain cases, esophageal diversion, with or without esophageal resection as appropriate. Determining the optimal therapy for our patients requires examining all available options, including surgery alone, endoscopic therapy alone or a hybrid combination of the two. palliative stent and two surgically). Primary endotherapy was performed for eight (50\%) and primary surgery for eight (50\%) patients. Two endotherapy patients required multiple stents. Median stent duration was 61 days (range 56-76). "Salvage" intervention was required in $2 / 8$ (25\%) endotherapy patients and $1 / 8(13 \%)$ surgery patient (stent). All patients healed without resection/reconstruction. There were no deaths in the surgically treated group and two in the endotherapy group (stented with palliative intent due to poor systemic condition). Readmission within 30 days occurred in $3 / 6$ of alive endotherapy patients (50\%) and 0/8 surgery patients. Re-intervention within 30 days was required for one endotherapy patient.

Conclusion: Endoscopic repair of Boerhaave perforations can be useful in carefully selected patients without evidence of systemic sepsis. Endoscopic therapy such as stenting is particularly valuable as a "salvage" intervention. The benefits of endoscopic therapy and esophageal preservation are offset against an increased risk of readmission in patients primarily treated endoscopically.

Mortality from esophageal perforation is high and attempts to reduce this are important. One proposed method is to use endoluminal approaches for the treatment of these perforations, in combination with adequate nutrition and appropriate drainage. Recent reviews and meta-analyses examining treatment of esophageal perforations have demonstrated that mortality rates may be lower when stents are used, although there is likely bias in the selection of these patients $[1,2]$. The use of endoluminal therapies includes endoluminal suturing (OverStitch, Apollo Endosurgery Inc., Austin, TX, United States), through-the-scope (TTS) clips, and over-the-scope clips (OTSC, Ovesco Inc., Tubingen, Germany). Success with regard to the use of these endoscopic devices has been reported in the context of treating gastrointesti- 
nal perforations [3-7]. Endoscopic suturing of a stent in the esophagus when there is no stricture present, such as in the context of a spontaneous perforation, has been reported to successfully anchor the stent and minimize the risk of migration [7]. With the availability and use of endoluminal therapies [8], it is important to assess their utility in comparison to surgical intervention for this acute condition. Any benefit of a less invasive endoscopic approach in terms of leak healing, need for "salvage" intervention, and mortality would be important for appropriate patient selection.

The aim of this study was to evaluate outcomes for patients with Boerhaave esophageal perforation who were treated surgically and with endoscopic therapy. Our aim was to determine the utility of endoscopic therapy in the management of these patients in order to guide future treatment algorithms.

\section{Patients and methods}

\section{$\nabla$}

We identified all adult patients ( $>18$ years) who were diagnosed with a spontaneous esophageal perforation over a 10 -year period (1 October 2004-1 October 2014). We excluded all patients who had been diagnosed and treated elsewhere before transfer to our institution. Those patients who did not consent to their data being used in the study were also excluded. Spontaneous perforations (Boerhaave perforation) were defined as perforation of a normal esophagus without previous instrumentation, surgery, or trauma. Data were collected from prospectively maintained databases. These data included: patient demographics, comorbidities, the intended treatment (conservative, endoscopic, surgical or a combination thereof), need for "salvage" intervention (defined as intervention intended to heal the perforation or treat initial sepsis), persistence of leak at discharge, discharge location, alimental status at discharge, discharge on antibiotics or drains in situ, readmission to our institution within 30 days, re-intervention within 30 days, survival at 30 days, and long-term follow-up. For those patients who underwent endoscopic therapy, the modalities used were through-the-scope and over-the-scope clips, endoscopic suturing (using the Apollo device), and esophageal stenting. To enable endoscopic suturing, an overtube was placed to avoid esophageal injury. A dual-channel endoscope was inserted through this to the site of the perforation. Using the suturing device, suitable defects were closed in either an interrupted or continuous fashion with 2-0 polypropylene or 2-0 polydioxanone sutures. A tissue helix was used at times to grip the esophageal mucosa and facilitate suturing.

\section{Results}

$\nabla$

A total of 17 patients with spontaneous esophageal perforation were identified. Sixteen patients consented to their data being used in the study. The median age at perforation was 68 years (range 27-88 years). Thirteen patients (81\%) underwent intervention for their perforation within 24 hours of their diagnosis. The most common investigation used to diagnose perforation was CT (63\% patients), followed by upper gastrointestinal endoscopy (25\%) and upper gastrointestinal swallow study (12\%). All patients ultimately underwent upper gastrointestinal endoscopy to confirm the diagnosis even if the diagnosis was made with another modality.
Endoscopic therapy was the primary intervention in eight patients (50\%) whereas surgery was the primary treatment modality in eight patients (50\%). Patients were offered primary treatment with endoluminal therapy from July 2010 onwards.

Of the eight patients who underwent endoluminal intervention, the median size of perforation was $1 \mathrm{~cm}$ (range $0.8-3 \mathrm{~cm}$ ). Two patients were stented with palliative intent and were septic and shocked at the time of presentation. The remaining six patients had no signs of sepsis. Two patients with small perforations were treated successfully with through-the-scope or over-thescope clips alone (Ovesco: $0.8 \mathrm{~cm}$ perforation, Resolution clip: 1 $\mathrm{cm}$ perforation). Six patients had stent insertion to treat their esophageal perforation, median size $1 \mathrm{~cm}$ (range $1-3 \mathrm{~cm}$ ). Of these six patients, two underwent attempted closure of the perforation using the Apollo Overstitch device (one defect completely closed, the other failed). One patient had the defect partially closed with an Ovesco clip, then required endosuturing to complete the closure before the stent was finally deployed. Another patient had the defect partially closed with through-the-scope clips before stent deployment. All of the stented patients underwent endoluminal stent fixation: 3/6 were fixed with throughthe-scope clips and 3/6 with endosutures. The clinical progress of patients is charted in 1 Fig. 1.

"Salvage" intervention was required for $2 / 8$ endotherapy and $1 / 8$ surgical patients ( $\bullet$ Fig. 1). Salvage intervention involved endoluminal therapy in all three patients ( $\bullet$ Table 1$)$. Median lengths of stay (LOS) were 26.5 days (range 7-80) and 9 days (range 542) for the surgery and endotherapy groups, respectively ( $P=$ 0.04). We excluded from this analysis the two patients in the endotherapy group who died in hospital. Five patients in the surgery group (63\%) and three patients in the endotherapy group (50\%) were discharged home. One patient in each group was discharged systemically well but with a persistent leak. Three patients in the surgery group $(3 / 8 ; 38 \%)$ and two patients in the endotherapy group $(2 / 6 ; 33 \%)$ were discharged home with drains. One patient in the surgery group (13\%) and three patients in the endotherapy group (50\%) were discharged on antibiotics. Six patients in the surgery group (75\%) and four patients in the endotherapy group (67\%) were discharged with enteral nutrition. More patients in the endotherapy group were discharged NPO (nothing by mouth) (3/8 surgery, 38\% v 4/6 endotherapy, 67\%). Two patients in the stented group died in hospital. These patients were considered unfit for surgery before any intervention. Readmission within 30 days occurred in $3 / 6$ and in $0 / 8$ patients in the endotherapy and surgical groups, respectively. Reasons for readmission included blocked feeding tube, dysphagia after resuming oral intake, and dislodged chest tube and feeding jejunostomy tube in association with sepsis. One diabetic patient in the surgery group with Boerhaave syndrome related to hyperemesis gravidarum required multiple stricture dilatations for dysphagia. She subsequently developed a fistula and paraesophageal abscess. Her initial surgery was a left thoracotomy, repair of esophageal perforation and pleural flap.

\section{Discussion}

$\nabla$

Spontaneous esophageal perforation is a rare but life threatening pathology. We have identified 17 patients with this clinical problem treated at a tertiary care center over a 10 -year period which correlates with other series in comparable centers [10-12]. Minimally invasive surgical intervention is increasing, as is endo- 
Patients with spontaneous esophageal perforation $(n=16)$
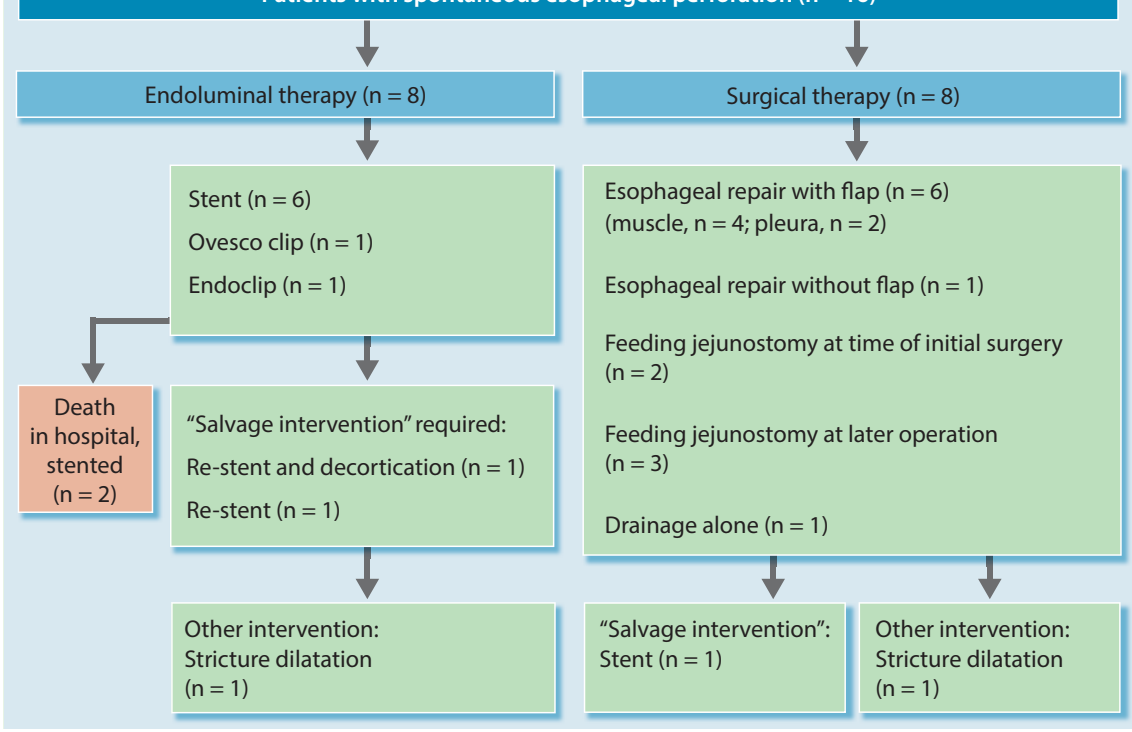

Fig. 1 Flowchart detailing the treatment options and outcomes for patients with esophageal perforations observed in our cohort. therapy for the treatment of many gastrointestinal pathologies, including esophageal perforation and esophageal anastomotic leak. Endotherapy for spontaneous esophageal perforation and subsequent leaks includes management with through-the-scope and over-the-scope clips, sutures or stents [13-15] ( Fig.2a, $\bullet$ Fig. 2 b, $\odot$ Fig. 2 c, $\odot$ Fig. 2 d, $\odot$ Fig. 2 e). In this study, size of perforation and the absence of sepsis determined the success of clips or stents (with endoluminal closure of the perforation) as primary therapy.

The principles of management of the esophageal perforation, however, have not changed. The need for sepsis control, repair of the perforation, feeding via a jejunostomy tube, and venting of gastric contents remains. When endotherapy is used to seal the perforation, stenting across the gastroesophageal junction does increase the risk of a life threatening aspiration. The availability of stents with one-way anti-reflux valves (EndoMAXX EVT, Merit, Salt Lake City, Utah, United States) has the potential to eliminate this specific adverse event. Until these are in wide- spread clinical use, jejunostomy feeding and gastric decompression are recommended to avoid this.

Patients with spontaneous esophageal perforation exhibit a spectrum of severity. Patients with significant pleural and mediastinal contamination are at high risk for sepsis and systemic inflammatory response syndrome (SIRS). Accordingly, based on this experience, we suggest the following endoscopic approach: for patients presenting early ( $<24$ hours) with a defect of $\leq 1 \mathrm{~cm}$, closure with a through-the-scope or over-the-scope clip or suture. In the presence of extravasation of contrast from the esophagus or pleural effusion, empyema or mediastinal contamination, radiological or surgical (video-assisted thoracoscopic surgery (VATS) or thoracotomy) adjunctive drainage is performed. For those patients with a large defect $\geq 1 \mathrm{~cm}$, primary endoluminal closure can be attempted combined with stent placement, with pleural drainage as needed. Our experience identified patients with large perforations as having a need for eventual decortication. Surgery, thoracoscopic or open, should include drainage of sepsis, repair of the defect, and a muscle flap to cover this repair,

Table 1 Salvage endotherapy for failed earlier intervention.

\begin{tabular}{|c|c|c|c|}
\hline Patient & Initial intervention & Salvage intervention & Outcome \\
\hline 71-year-old man & $\begin{array}{l}\text { Esophageal perforation closed } \\
\text { with both OTSC and suturing with } \\
\text { Apollo endosuturing device. } \\
\text { A } 12.5-\mathrm{cm} \times 23 \mathrm{~mm} \text { FCSEMS was } \\
\text { also placed. }\end{array}$ & $\begin{array}{l}\text { Day 12: Left VATS decortication and chest } \\
\text { drain insertion for empyema. } \\
\text { Day } 28 \text { : Overlapping stents } \\
(12.5 \mathrm{~cm} \times 23 \mathrm{~mm}) \text {. } \\
\text { Day } 65 \text { : Stents removed. Persistent leak. } \\
\text { A } 15.5 \mathrm{~cm} \times 23 \mathrm{~mm} \text { PCSEMS was placed. }\end{array}$ & $\begin{array}{l}\text { Day 91: Stent in stent removal of stents. } \\
\text { No leak. }\end{array}$ \\
\hline 66-year-old woman & $\begin{array}{l}\text { Esophageal perforation closed } \\
\text { with TTS clips. A } 12 \mathrm{~cm} \times 23 \mathrm{~mm} \\
\text { PCSEMS was also placed and TTS } \\
\text { clips used to anchor the stent. }\end{array}$ & $\begin{array}{l}\text { Day 78: Stent removed-persistent fistula. } \\
\text { Suture closure of the fistula was performed } \\
\text { and a } 10.3 \mathrm{~cm} \times 23 \mathrm{~mm} \text { PCSEMS placed. }\end{array}$ & $\begin{array}{l}\text { Day 111: Stent within a stent (FCSEMS). } \\
\text { Day 129: Stents removed. } \\
\text { Persistent fistula-thermal preparation (APC) } \\
\text { with suture closure. } \\
\text { Subsequent stricture - dilatations required. }\end{array}$ \\
\hline 75-year-old woman & $\begin{array}{l}\text { Left thoracotomy, repair of } \\
\text { esophageal perforation and } \\
\text { intercostal muscle flap. } \\
\text { Day 13: Feeding jejunostomy. }\end{array}$ & $\begin{array}{l}\text { Day 20: Persistent leak. } \\
\text { Endoscopic assessment of the leak and clo- } \\
\text { sure using TTS clips and a } 12.3 \mathrm{~cm} \times 18 \mathrm{~mm} \\
\text { FCSEMS. The stent was sutured to anchor it in } \\
\text { place. }\end{array}$ & Day 76: Stent removed, no leak. \\
\hline
\end{tabular}

APC, argon plasma coagulation; OTSC, over-the-scope clip; TTS, through-the-scope; VATS, video assisted thoracoscopic surgery; FCSEMS, fully covered self-expanding metal stent; PCSEMS, partially covered self-expanding metal stent. 


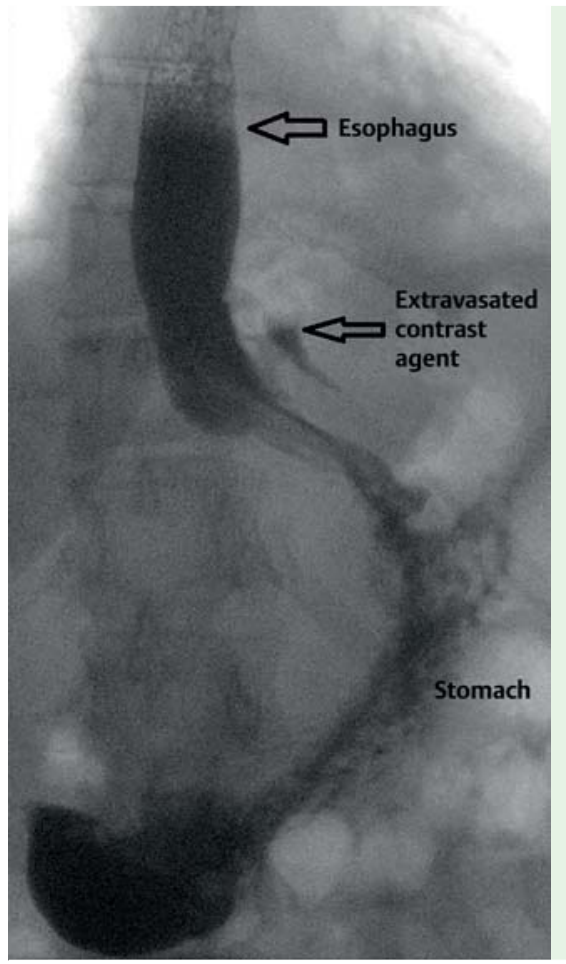

Fig. 2 a Contrast esophagram demonstrating a Boerhaave esophageal perforation.

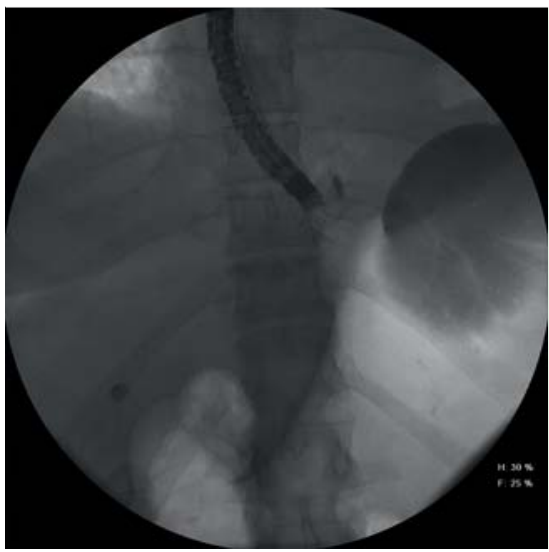

Fig. 2 b Combined radiological and endoscopic assessment of the perforation.

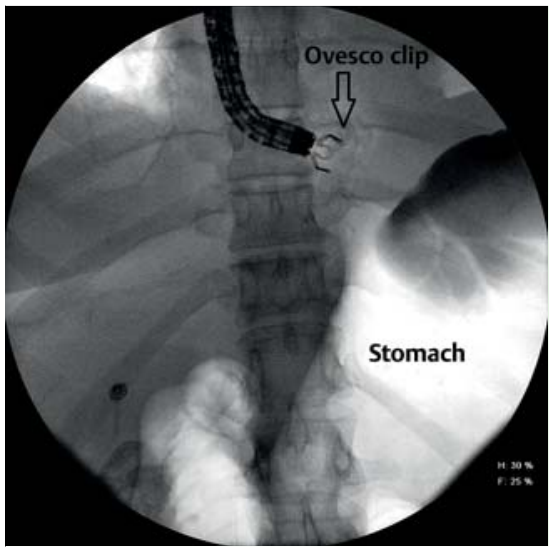

Fig. 2 c Endoscopic and radiological guidance to position the Ovesco over-the-scope clip to close the perforation.

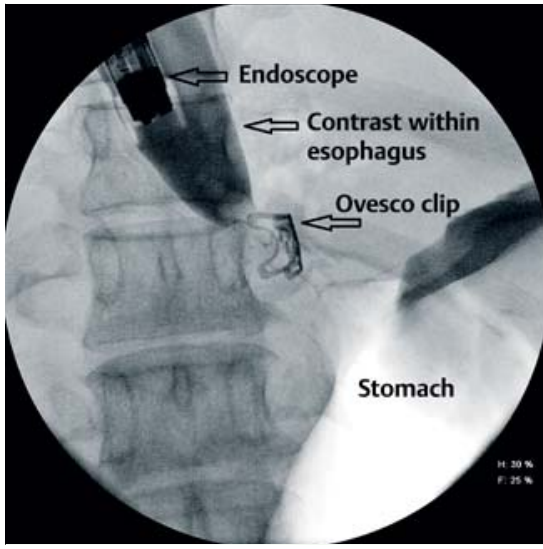

Fig. 2 d Contrast esophagram demonstrating no further esophageal leak after placement of the Ovesco clip.

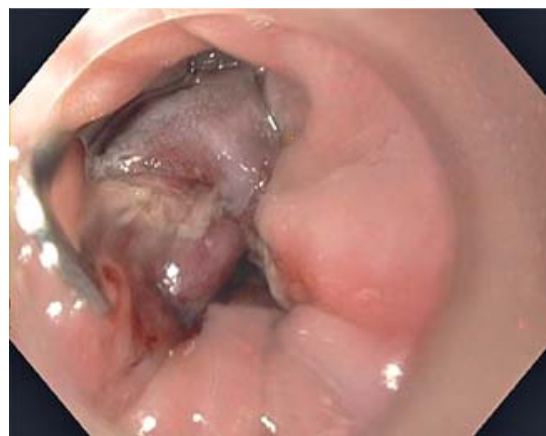

Fig. 2 e Endoscopic appearance of the sealed esophageal perforation using an Ovesco clip. in addition to a feeding tube (jejunostomy) and venting gastrostomy. For patients who have lost more than $50 \%$ of the esophageal diameter due to a more severe tear, delay in treatment or inadequate drainage, esophageal diversion or exclusion become major management considerations.

Salvage intervention for patients in this study always involved endoluminal therapy. One patient with a persistent leak after surgery required stenting and two stented patients required restenting. Stenting is preferable to clipping or suturing in this situation as the tissue is suboptimal for these closure methods. In the absence of sepsis in those treated primarily with early endoluminal therapy, further endoluminal therapy is desirable for leak control and minimization of sepsis risk. In post-surgical patients, re-intervention can be challenging, which makes an endoluminal option appealing as long as sepsis is well controlled.

$\mathrm{CT}$ was the most common diagnostic tool used to detect a leak. This is favored over a contrast swallow because of the additional information that can be provided, e.g. extent of any collections and presence of mediastinal contamination. All patients underwent subsequent upper gastrointestinal endoscopy after diagnosis (if this was not the diagnostic modality). This is essential in or- der to define the anatomy of the esophageal perforation and guide treatment.

Median length of stay was lower in the endotherapy group, while readmission was higher at $50 \%$. Patients who had the least contamination and physiological insult were those selected for primary endotherapy. Patients undergoing endotherapy also spent a greater proportion of their recovery out of hospital which may account for the increased readmission rates in this group. Of those patients who were readmitted after endotherapy, only one patient stayed in hospital for more than 2 days. Our surgical inhospital and 30-day mortality rate in patients treated with curative intent was $0 \%$. In the endotherapy group, two patients died, but those patients were treated with palliative intent.

The median duration that stents were in place was 59.5 days (range 23-76). During this time, two patients required multiple stents to be removed and replaced. Suturing for stent fixation has been supported in the literature and applied to our patients. Use of the current suturing device for this application in order to reduce stent migration is not formally approved in the FDA clearance. Although our small series showed no esophago-aortic fistulas, after review of the published literature [16-21], there is a 
well-recognized risk for this complication. Improved stent management may be able to reduce this risk and will require more formal study which may examine stent sizes, length of dwell time, stent free periods, and stent exchange.

With a paucity of Level 1 evidence, the expert consensus opinion from both our thoracic surgery division and the gastroenterology division favors a shorter duration of stenting and, if the leak is still present at the time of removal, use of a new stent that is of different length, size, or landing zone is encouraged. Aortic erosions do occur, and most appear to occur at the flare of the stent or at the location of perforation where the stent is in direct contact with the wall of the vessel. It is for this reason that we also recommend a muscle flap to interpose when a leak directly abuts the aorta or tracheobronchial tree.

In conclusion, our 10-year experience demonstrates that endoscopic repair of Boerhaave perforations can be useful in carefully selected patients without evidence of systemic sepsis. We have also found that endoscopic therapy such as stenting is particularly valuable as a "salvage" intervention regardless of whether the primary intervention was endoscopic or surgical. The benefits of endoscopic therapy and esophageal preservation may be offset against an increased risk of readmission in those patients primarily treated endoscopically. Our experience has shown that it is possible to manage this life-threatening pathology with surgery and endotherapy with low mortality.

\section{Competing interests: None}

\section{Acknowledgments}

$\nabla$

We acknowledge that the images used in this paper are modifications of those used in "Atlas of Esophageal Disease and Intervention: A Multidisciplinary Approach," Blackmon SH, Kim MP, Dickinson KJ, eds. Springer, November 2015.

\section{References}

1 Biancari F, D'Andrea V, Paone R et al. Current treatment and outcome of esophageal perforations in adults: systematic review and metaanalysis of 75 studies. World J Surg 2013; 37: 1051-1059

2 Dasari BV, Neeley D, Kennedy $A$ et al. The role of esophageal stents in the management of esophageal anastomotic leaks and benign esophageal perforations. Ann Surg 2014; 259: 852-860

3 Raju GS, Shibukawa G, Ahmed I et al. Endoluminal suturing may overcome the limitations of clip closure of a gaping wide colon. Gastrointest Endosc 2007; 65: 906 -911

4 Pham BV, Raju GS, Ahmed I et al. Immediate endoscopic closure of colon perforation by using a prototype endoscopic suturing device: feasibil- ity and outcome in a porcine model. Gastrointest Endosc 2006; 64: $113-119$

5 Wasano K, Hashiguchi S, Suzuki N et al. Transoral closure of pharyngeal perforation caused by gastrointestinal endoscopy. Auris Nasus Larynx 2014; 41: 113-117

6 Schaheen L, Blackmon SH, Nason KS. Optimal approach to the management of intrathoracic esophageal leak following esophagectomy: a systematic review. Am J Surg 2014; 208: 536-543

7 Stephens EH, Correa AM, Kim MP et al. Classification of esophageal stent leaks: leak presentation, complications and management. Ann Thorac Surg 2014; 98: 297 -304

8 Fujii LL, Bonin EA, Baron TH et al. Utility of an endoscopic suturing system for the prevention of covered luminal stent migration in the upper GI tract. Gastrointest Endosc 2013; 78: 787-793

9 Kumta NA, Boumitri C, Kahaleh M. New devices and techniques for handling adverse events: claw, suture or cover? Gastrointest Endosc Clin N Am 2015; 25: 159-168

10 Lindenmann J, Matzi V, Neuboeck $N$ et al. Management of esophageal perforation in 120 consecutive patients: clinical impact of a structured treatment algorithm. J Gastrointest Surg 2013; 17: 1036-1043

11 Sulpice L, Dileon S, Rayar M et al. Conservative surgical management of Boerhaave's syndrome: experience of two tertiary referral centers. Int J Surg 2013; 11: 64-67

12 Cho JS, Kim YD, Kim JW et al. Thoracoscopic primary esophageal repair in patients with Boerhaave's syndrome. Ann Thorac Surg 2011; 91: $1552-1555$

13 Musala C, Eisendrath P, Brasseur A et al. Successful treatment of Boerhaave syndrome with an over the scope clip. Endoscopy 2015; 47: E24-5

14 Van Weyenberg SJ, Stam FJ, Marsman W. Successful endoscopic closure of spontaneous esophageal rupture (Boerhaave syndrome). Gastrointest Endosc 2014; 80: 162

15 Kobara H, Mori H, Rafiq K et al. Successful endoscopic treatment of Boerhaave syndrome using an over-the-scope clip. Endoscopy 2014; 46: E82-83

16 D'Cuhna J, Rueth NM, Groth SS et al. Esophageal stents for anastomotic leaks and perforations. J Thorac Cardiovasc Surg 2011; 142: 39-46

17 Han XW, Li YD, Wu G et al. New covered mushroom shaped metallic stent for managing anastomotic leak after esophagogastrostomy with a wide gastric tube. Ann Thorac Surg 2006; 82: 702 - 706

18 Jiang $F, Y u M F$, Ren $B H$ et al. Nasogastric placement of a sump tube through the leak for the treatment of esophagogastric anastomotic leak after esophagectomy for esophageal carcinoma. J Surg Res 2011; 171: $448-451$

19 Kim AW, Liptay MJ, Snow N et al. Utility of silicone esophageal bypass stents in the management of delayed complex esophageal disruptions. Ann Thorac Surg 2008; 85: 1962-1967

20 Pennathur A, Chang AC, McGrath KM et al. Polyflex expandable stents in the treatment of esophageal disease: initial experience. Ann Thorac Surg 2008; 85: $1968-1972$

21 Schweigert M, Dubecz A, Stadlhuber RJ et al. Risks of stent related aortic erosion after endoscopic stent insertion for intrathoracic anastomotic leaks after esophagectomy. Ann Thorac Surg 2011; 92: 513-518 